OPEN ACCESS

Edited by:

Srilakshmi Srinivasan, Queensland University of Technology, Australia

Reviewed by: Sumit Isharwal, University of Virginia, United States

Piotr Bryniarski,

Medical University of Silesia, Poland

*Correspondence: Mike Wenze Mike.Wenzel@kgu.de

Specialty section: This article was submitted to Genitourinary Oncology, a section of the journal

Frontiers in Oncology

Received: 07 August 2021 Accepted: 20 September 2021 Published: 06 October 2021

Citation:

Wenzel M, Nocera L, Würnschimmel C, Collà Ruvolo C, Tian Z, Saad F, Briganti A, Tilki D, Graefen M, Becker A, Roos FC, Chun FKH and Karakiewicz PI (2021) The Effect of 10 Most Common Nonurological Primary Cancers on Survival in Men With Secondary Prostate Cancer.

Front. Oncol. 11:754996. doi: 10.3389/fonc.2021.754996

\section{The Effect of 10 Most Common Nonurological Primary Cancers on Survival in Men With Secondary Prostate Cancer}

Mike Wenzel ${ }^{1,2 *}$, Luigi Nocera ${ }^{2,3}$, Christoph Würnschimmel ${ }^{2,4}$, Claudia Collà Ruvolo ${ }^{2,5}$, Zhe Tian $^{2}$, Fred Saad ${ }^{2}$, Alberto Briganti ${ }^{3}$, Derya Tilki ${ }^{4,6}$, Markus Graefen $^{4}$, Andreas Becker ${ }^{1}$, Frederik C. Roos ${ }^{1}$, Felix K. H. Chun ${ }^{1}$ and Pierre I. Karakiewicz ${ }^{2}$

${ }^{1}$ Department of Urology, University Hospital Frankfurt, Frankfurt am Main, Germany, ${ }^{2}$ Cancer Prognostics and Health Outcomes Unit, Division of Urology, University of Montréal Health Center, Montréal, QC, Canada, ${ }^{3}$ Department of Urology and Division of Experimental Oncology, Urological Research Institute (URI), Istituto di Ricovero e Cura a Carattere Scientifico (IRCCS) San Raffaele Scientific Institute, Milan, Italy, ${ }^{4}$ Martini-Klinik Prostate Cancer Center, University Hospital HamburgEppendorf, Hamburg, Germany, ${ }^{5}$ Department of Neurosciences, University of Naples Federico II Reproductive Sciences and Odontostomatology, Naples, Italy, ${ }^{6}$ Department of Urology, University Hospital Hamburg-Eppendorf, Hamburg, Germany

Background: This study aims to test the effect of the 10 most common nonurological primary cancers (skin, rectal, colon, lymphoma, leukemia, pancreas, stomach, esophagus, liver, lung) on overall mortality (OM) after secondary prostate cancer (PCa).

Material and Methods: Within the Surveillance, Epidemiology, and End Results (SEER) database, patients with 10 most common primary cancers and concomitant secondary PCa (diagnosed 2004-2016) were identified and were matched in 1:4 fashion (age, year at diagnosis, race/ethnicity, treatment type, TNM stage) with primary PCa controls. OM was compared between secondary and primary PCa patients and was stratified according to primary cancer type, as well as according to time interval between primary cancer vs. secondary PCa diagnoses.

Results: We identified 24,848 secondary PCa patients (skin, $n=3,871$; rectal, $n=798$; colon, $n=3,665$; lymphoma, $n=2,583$; leukemia, $n=1,102$; pancreatic, $n=118$; stomach, $n=361$; esophagus, $n=219$; liver, $n=160$; lung, $n=1,328)$ vs. 531,732 primary PCa patients. Secondary PCa characteristics were less favorable than those of primary PCa patients (PSA and grade), and smaller proportions of secondary PCa patients received active treatment. After 1:4 matching, all secondary PCa exhibited worse OM than primary PCa patients. Finally, subgroup analyses showed that the survival disadvantage of secondary PCa patients decreased with longer time interval since primary cancer diagnosis and subsequent secondary PCa. 


\begin{abstract}
Conclusion: Patients with secondary PCa are diagnosed with less favorable PSA and grade. Even after matching for PCa characteristics, secondary PCa patients still exhibit worse survival. However, the survival disadvantage is attenuated, when secondary PCa diagnosis is made after longer time interval, since primary cancer diagnosis.
\end{abstract}

Keywords: mortality, primary prostate cancer, lung cancer, colon cancer, secondary cancer

\section{INTRODUCTION}

The most recent US cancer statistics (2018) indicate over 17 million new cancer diagnoses annually. Of these, almost 9 million were made in men (1-3). In men, prostate cancer (PCa) ranks as first or second most frequently diagnosed cancer. Virtually, all contemporary epidemiological studies addressing PCa survival exclusively focused on primary PCa and excluded patients with prior cancers (4-9). It is particularly of note that an increased risk exists for secondary cancers and especially secondary PCa after prior primary cancers (10-16). However, only three epidemiological SEER-based studies ( $n=18,225 ; n=5,987 ; n=1,457)$ and one European institutional study $(n=1,552)$ addressed mortality in patients with secondary $\mathrm{PCa}$, after initial diagnosis of another malignancy (17-20). All three studies showed worse survival in secondary PCa patients, relative to primary PCa patients. However, none stratified their analyses according to the most common cancer types. However, primary skin cancer may have a different effect than lung cancer. Moreover, it may also be postulated that the time interval between primary cancer and secondary $\mathrm{PCa}$ diagnosis may also affect survival in secondary PCa patients but has not been examined to date.

We addressed these two important unaddressed points within the Surveillance, Epidemiology, and End Results (SEER) registry database and hypothesized that they may impact important survival differences.

\section{MATERIAL AND METHODS}

\section{Study Population}

Within the SEER database, we identified all patients $\geq 18$ years old with secondary PCa diagnosed between 2004 and 2016, after prior diagnosis of one of 10 commonest nonurological malignancies (skin, rectal, colon, lymphoma, leukemia, pancreas, stomach, esophagus, liver, and lung). Moreover, we also identified all $\geq 18$-year-old patients with biopsy-proven primary adenocarcinoma of the prostate diagnosed between 2004 and 2016 (International Classification of Disease for Oncology (ICD-O-3) code 8140, site code C61.9). Cases that were identified at autopsy or death certificate or with unknown histology were excluded. Patients with unavailable PSA values were excluded in both cohorts. We excluded concomitantly diagnosed primary cancer and secondary PCa ( $\leq 6$ months apart), according to previously reported methodology $(21,22)$. Descriptive statistics addressed all included 24,848 secondary $\mathrm{PCa}$ patients and all 531,732 primary PCa patients
(Figure 1; Table 1). Subsequently, survival analyses focused on overall mortality (OM). Here, we relied on a propensity score matched (age at diagnosis, year of diagnosis, race/ethnicity, PCa treatment, cT-stage, cN-stage, and M-stage) cohort of all 24,848 secondary PCa patients that were matched with four primary PCa controls $(n=99,392)$.

\section{Statistical Analysis}

Descriptive statistics included frequencies and proportions for categorical variables. Medians and interquartile ranges (IQR) were reported for continuously coded variables. The Chi-square tested the statistical significance in proportion differences. The $t$ test and Kruskal-Wallis test examined the statistical significance of mean and distribution differences.

The first part of the analyses compared patient and PCa characteristics between all identified secondary $(n=24,848)$ and primary PCa patients $(n=531,732)$. In the second part of the analyses, we focused on overall mortality (OM), after 1:4 propensity score matching. Kaplan-Meier illustrated OM in the overall comparisons, as well as in all subsequent subgroup analyses. Additionally, multivariable Cox regression quantified hazard ratios (HR) that compared secondary vs. primary $\mathrm{PCa}$ patients, after further adjusting for covariates of the 1:4 matched cohort: PSA, socioeconomic status, Gleason grade group, and D'Amico risk group (all not previously matched). All tests were two sided with a level of significance set at $p<0.05$ and $\mathrm{R}$ software environment for statistical computing and graphics (version 3.4.3) was used for all analyses (23).

\section{RESULTS}

\section{Descriptive Characteristics of the Study Population Prior to Matching}

Prior to matching, 24,848 secondary PCa and 531,732 primary PCa were available for analyses (Table 1). Patients with secondary PCa more frequently harbored Gleason grade group IV $(10.3 \%$ vs. $8.8 \%)$ and V (9.3\% vs. $7.7 \%, p<0.001)$. Median PSA at diagnosis showed marginal differences between secondary and primary PCa patients (6.9 [IQR 4.9-11.5] vs. $6.6 \mathrm{ng} / \mathrm{ml}$ [IQR 4.8-10.6], $p<0.001)$. In secondary PCa patients, median PSA values at diagnosis of secondary PCa ranged from 6.5 (skin cancer) to $7.8 \mathrm{ng} / \mathrm{ml}$ (pancreatic and liver cancer). However, median age at secondary $\mathrm{PCa}$ diagnosis was more advanced than in primary PCa (69 vs. 65 years, $p<0.001)$. In secondary PCa patients (Table 2), median age at secondary PCa diagnoses ranged from respectively 66 (liver cancer) to 72 years (colon cancer). The average time interval between primary 


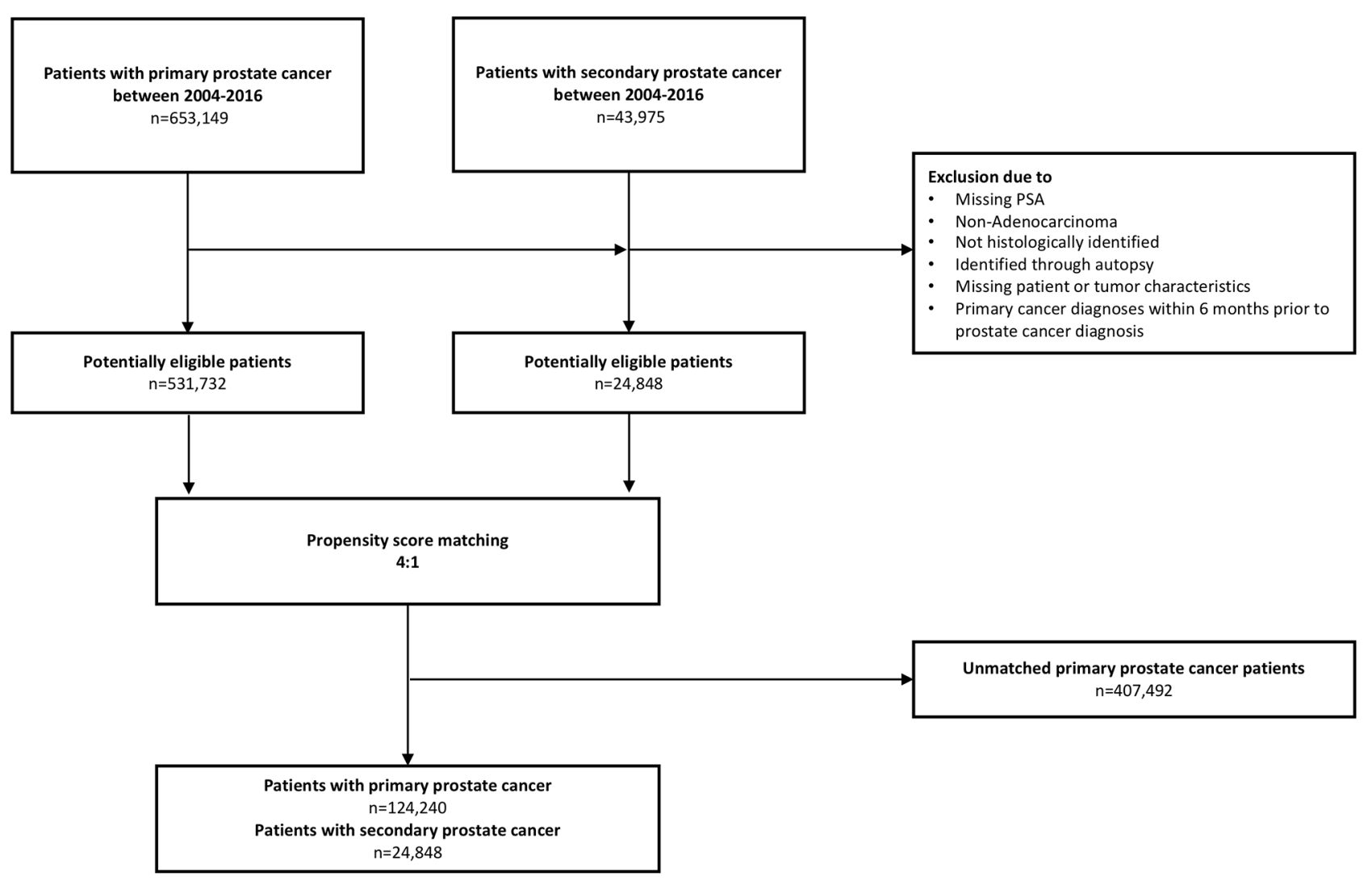

FIGURE 1 | Flow chart depicting included patients with primary and secondary prostate cancer in analyses.

cancer diagnosis and secondary PCa diagnosis ranged from 5 (pancreatic, esophagus, liver cancer) to 8 years (skin and rectum cancer). No clinically meaningful differences were recorded in cT-stage, cN-stage, and M-stages between secondary and primary PCa patients. Important differences existed according to use of local therapy [external beam radiation therapy (EBRT) and radical prostatectomy (RP)]. Specifically, in secondary PCa patients, the rate of EBRT was higher (25.7\% vs. 22.7\%) and the rate of $\mathrm{RP}$ was lower (23.8\% vs. $33.5 \%)$, relative to primary $\mathrm{PCa}$ patients (all $p<0.001$ ). In secondary PCa patients, rates of RP ranged from $11.3 \%$ (liver cancer) to $29.7 \%$ (skin cancer) and rates of EBRT ranged from $19.5 \%$ (rectal cancer) to $34.4 \%$ (liver cancer).

\section{Survival Analyses After 1:4 Propensity Score Matching}

After matching, OM at 10 years was $46.0 \%$ in secondary PCa vs. $35.7 \%$ in primary $\mathrm{PCa}$ (Figure 2A). The median survival of all 24,848 secondary PCa patients was 131 months and not reached for 99,392 primary PCa patients. This survival disadvantage translated into a 1.49-fold higher risk of OM in secondary $\mathrm{PCa}$ patients, relative to their primary PCa counterparts. After further multivariable adjustment, a 1.51-fold higher $\mathrm{OM}$ was observed (Table 3).

\section{Survival Analyses After 1:4 Propensity Score Matching According to Local Treatment Type: RP vs. EBRT vs. No Local Treatment}

Subsequently, we repeated Kaplan-Meier and Cox regression analyses, after stratification according to local PCa treatment type in patients treated with RP or EBRT or no local treatment (NLT) across all primary cancer types. Here, presence of secondary $\mathrm{PCa}$ resulted in worse $\mathrm{OM}$, relative to primary $\mathrm{PCa}$ patients. Specifically, 10-year OM rates were respectively $22.1 \%$ vs. $11.7 \%, 47.4 \%$ vs. $36.5 \%$, and $75.3 \%$ vs. $51.7 \%$ after RP, EBRT, or NLT in secondary vs. primary PCa patients (Figures $2 \mathbf{B}-\mathbf{D}$ ). In multivariable Cox regression models, the respective HRs were 2.3 after RP, 1.6 after EBRT, and 1.5 after NLT in secondary PCa patients, relative to primary PCa patients (Table 3 , all <0.01).

\section{Survival Analyses After 1:4 Propensity Score Matching According to Primary Cancer Type}

Kaplan-Meier plots showed in secondary PCa patients with skin, rectal, pancreas, colon, lymphoma, leukemia, stomach, liver, esophagus, and lung cancer $v s$. for primary PCa patients respectively 10 -year OM rates of $33.6 \%$ vs. $32.1 \%, 43.7 \%$ vs. $39.3 \%, 45.7 \%$ vs. $32.2 \%, 46.4 \%$ vs. $41.7 \%, 49.3 \%$ vs. $34.8 \%, 52.9 \%$ 
TABLE 1 | Descriptive characteristics prior to matching and after matching for age at prostate cancer diagnosis, year of prostate cancer diagnoses, race/ethnicity, treatment type, and TNM stage for primary and secondary prostate cancer patients.

\begin{tabular}{|c|c|c|c|c|c|c|}
\hline \multirow[t]{2}{*}{ Variable } & & \multicolumn{2}{|c|}{ Prior to matching } & \multicolumn{3}{|c|}{ After matching } \\
\hline & & $\begin{array}{l}\text { Primary PCa } \\
N=531,732\end{array}$ & $\begin{array}{c}\text { Secondary PCa } \\
N=24,848\end{array}$ & $\begin{array}{c}\text { Overall } \\
N=124,240\end{array}$ & $\begin{array}{c}\text { Primary PCa } \\
N=99,392(80 \%)\end{array}$ & $\begin{array}{l}\text { Secondary PCa } \\
N=24,848(20 \%)\end{array}$ \\
\hline Age at PCa diagnosis & Median (IQR) & $65(59-72)$ & $69(64-76)$ & $69(64-76)$ & $69(64-76)$ & $69(64-76)$ \\
\hline Year of PCa diagnosis & Median (IQR) & 2010 (2007-2013) & $2013(2007-2013)$ & 2013 (2007-2013) & 2013 (2007-2013) & 2013 (2007-2013) \\
\hline Age of primary cancer diagnosis & Median (IQR) & - & $63(56-69)$ & - & - & $63(56-69)$ \\
\hline Year of primary cancer diagnosis & Median (IQR) & - & 2004 (2000-2008) & - & - & 2004 (2000-2008) \\
\hline PSA (ng/ml) & Median (IQR) & $6.6(4.8-10.6)$ & $6.9(4.9-11.5)$ & $6.9(4.9-11.4)$ & $6.9(4.9-11.3)$ & $6.9(4.9-11.5)$ \\
\hline Follow-up (months) & Median (IQR) & $68(32-104)$ & $53(23-88)$ & $58(25-93)$ & $59(26-94)$ & $53(23-88)$ \\
\hline \multirow[t]{5}{*}{ cT } & cT1 & 324,967 (61.1) & $14,719(59.2)$ & $74,330(59.8)$ & $59,611(60.0)$ & $14,719(59.2)$ \\
\hline & сT2 & $164,054(30.9)$ & 7,919 (31.9) & $40,322(32.5)$ & 32,403 (32.6) & 7,919 (31.9) \\
\hline & сТ3 & $14,084(2.6)$ & $635(2.6)$ & $2,853(2.3)$ & $2,218(2.2)$ & $635(2.6)$ \\
\hline & cT4 & $4,701(0.9)$ & $248(1.0)$ & $926(0.7)$ & $678(0.7)$ & $248(1.0)$ \\
\hline & cTx & $23,926(4.5)$ & $1,327(5.3)$ & $5,809(4.7)$ & 4,482 (4.5) & 1,327 (5.3) \\
\hline \multirow[t]{3}{*}{ cN stage } & cNO & 493,330 (92.8) & 23,026 (92.7) & 116,645 (93.9) & $93,619(94.2)$ & 23,026 (92.7) \\
\hline & cN1 & $15,055(2.8)$ & $573(2.3)$ & 2,295 (1.8) & $1,722(1.7)$ & $573(2.3)$ \\
\hline & $\mathrm{cNx}$ & $23,347(4.4)$ & $1,249(5.0)$ & $5,300(4.3)$ & $4,051(4.1)$ & $1,249(5)$ \\
\hline \multirow[t]{3}{*}{ M stage } & $\mathrm{MO}$ & 495,768 (93.2) & $23,021(92.6)$ & $116,431(93.7)$ & $93,410(94.0)$ & 23,021 (92.6) \\
\hline & M1 & 22,396 (4.2) & $1,131(4.6)$ & 4,834 (3.9) & $3,703(3.7)$ & $1,131(4.6)$ \\
\hline & $\mathrm{Mx}$ & $13,568(2.6)$ & $696(2.8)$ & $2,975(2.4)$ & 2,279 (2.3) & $696(2.8)$ \\
\hline \multirow[t]{6}{*}{ Gleason grade group at diagnosis } & 1 & $209,565(39.4)$ & $8,951(36.0)$ & $46,422(37.4)$ & $37,471(37.7)$ & $8,951(36.0)$ \\
\hline & $\|$ & 137,937 (25.9) & 6,117 (24.6) & $30,986(24.9)$ & $24,869(25.0)$ & $6,117(24.6)$ \\
\hline & III & 60,193 (11.3) & 2,968 (11.9) & $14,813(11.9)$ & 11,845 (11.9) & 2,968 (11.9) \\
\hline & IV & $46,788(8.8)$ & 2,548 (10.3) & $12,368(10.0)$ & $9,820(9.9)$ & 2,548 (10.3) \\
\hline & V & $40,687(7.7)$ & 2,299 (9.3) & 10,795 (8.7) & 8,496 (8.5) & 2,299 (9.3) \\
\hline & Unknown & $36,562(6.9)$ & $1,965(7.9)$ & $8,856(7.1)$ & $6,891(6.9)$ & $1,965(7.9)$ \\
\hline \multirow[t]{4}{*}{ D’Amico risk group } & low & 135,502 (25.5) & 5,538 (22.3) & 29,178 (23.5) & 23,640 (23.8) & 5,538 (22.3) \\
\hline & intermediate & $210,982(39.7)$ & 9,892 (39.8) & 49,444 (39.8) & 39,552 (39.8) & 9,892 (39.8) \\
\hline & high & 144,985 (27.3) & 7,319 (29.5) & $36,118(29.1)$ & 28,799 (29.0) & 7,319 (29.5) \\
\hline & Unknown & 40,263 (7.6) & 2,099 (8.4) & $9,500(7.6)$ & $7,401(7.4)$ & 2,099 (8.4) \\
\hline \multirow[t]{8}{*}{ Treatment } & $\mathrm{RP}$ & 178,084 (33.5) & 5,909 (23.8) & 29,099 (23.4) & 23,190 (23.3) & 5,909 (23.8) \\
\hline & EBRT & $120,891(22.7)$ & $6,377(25.7)$ & 32,032 (25.8) & 25,655 (25.8) & $6,377(25.7)$ \\
\hline & BT & 39,655 (7.5) & 1,718 (6.9) & 9,023 (7.3) & 7,305 (7.3) & 1,718 (6.9) \\
\hline & $\mathrm{BT}+\mathrm{EBRT}$ & $21,696(4.1)$ & 952 (3.8) & 4,755 (3.8) & 3,803 (3.8) & 952 (3.8) \\
\hline & $\mathrm{RP}+\mathrm{EBRT}$ & $15,121(2.8)$ & $554(2.2)$ & $2,684(2.2)$ & $2,130(2.1)$ & $554(2.2)$ \\
\hline & $\mathrm{RT}+\mathrm{RP}$ & $156(0)$ & $8(0)$ & $33(0)$ & $25(0)$ & $8(0)$ \\
\hline & NLT & $140,081(26.3)$ & $8,430(33.9)$ & $42,278(34.0)$ & $33,848(34.1)$ & $8,430(33.9)$ \\
\hline & Unknown & 16,048 (3.0) & 900 (3.6) & 4,336 (3.5) & $3,436(3.5)$ & 900 (3.6) \\
\hline \multirow[t]{2}{*}{ Chemotherapy } & No/Unknown & 527,509 (99.2) & 24,663 (99.3) & 123,432 (99.3) & 98,769 (99.4) & 24,663 (99.3) \\
\hline & Yes & 4,223 (0.8) & $185(0.7)$ & $808(0.7)$ & $623(0.6)$ & $185(0.7)$ \\
\hline \multirow[t]{6}{*}{ Race/ethnicity } & Caucasian & 363,223 (68.3) & 19,536 (78.6) & $97,760(78.7)$ & $78,224(78.7)$ & 19,536 (78.6) \\
\hline & African American & 81,905 (15.4) & $2,758(11.1)$ & $13,890(11.2)$ & $11,132(11.2)$ & $2,758(11.1)$ \\
\hline & Hispanic & $48,835(9.2)$ & $1,494(6.0)$ & $7,468(6.0)$ & $5,974(6.0)$ & $1,494(6.0)$ \\
\hline & Native & 1,861 (0.3) & $80(0.3)$ & $340(0.3)$ & $260(0.3)$ & $80(0.3)$ \\
\hline & Asian & $26,007(4.9)$ & $948(3.8)$ & $4,613(3.7)$ & $3,665(3.7)$ & $948(3.8)$ \\
\hline & Unknown & $9,901(1.9)$ & $32(0.1)$ & $169(0.1)$ & $137(0.1)$ & $32(0.1)$ \\
\hline \multirow[t]{3}{*}{ Marital status } & Married & 354,363 (66.6) & $17,024(68.5)$ & $82,781(66.6)$ & $65,757(66.2)$ & $17,024(68.5)$ \\
\hline & Unmarried & 116,788 (22.0) & 5,049 (20.3) & 26,519 (21.3) & $21,470(21.6)$ & 5,049 (20.3) \\
\hline & Unknown & $60,581(11.4)$ & 2,775 (11.2) & $14,940(12)$ & 12,165 (12.2) & 2,775 (11.2) \\
\hline \multirow[t]{2}{*}{ Socioeconomic status } & 1st quartile & $133,678(25.1)$ & 6,170 (24.8) & 32,867 (26.5) & 26,697 (26.9) & $6,170(24.8)$ \\
\hline & 2nd-4th quartile & $397,946(74.8)$ & $18,678(75.2)$ & $91,373(73.5)$ & 72,695 (73.1) & $18,678(75.2)$ \\
\hline \multirow[t]{4}{*}{ Region } & West & 270,363 (50.8) & $12,440(50.1)$ & $62,122(50)$ & $49,682(50)$ & $12,440(50.1)$ \\
\hline & Midwest & 51,705 (9.7) & 3,417 (13.8) & $13,753(11.1)$ & $10,336(10.4)$ & 3,417 (13.8) \\
\hline & North-East & 89,653 (16.9) & 4,363 (17.6) & 21,531 (17.3) & 17,168 (17.3) & 4,363 (17.6) \\
\hline & South & $120,011(22.6)$ & 4,628 (18.6) & 26,834 (21.6) & 22,206 (22.3) & 4,628 (18.6) \\
\hline
\end{tabular}

vs. $35.2 \%, 55.6 \%$ vs. $40.1 \%, 57.1 \%$ vs. $29.5 \%, 63.7 \%$ vs. $42.5 \%$, and 67.0\% vs. 37.9\% (Figures 3 and 4). All secondary PCa patients harbored a significant $\mathrm{OM}$ disadvantage relative to primary $\mathrm{PCa}$ patients. The specific multivariable HRs were $1.2,1.3,1.8,1.2$, $1,8,1.8,1.9,3.0,1.8$, and 2.5 for respectively secondary $\mathrm{PCa}$ patients with primary skin, rectal, pancreas, colon, lymphoma, leukemia, stomach, liver, esophagus, and lung cancer (all $p<0.01$; Table 3).

The proportions of patients that died of secondary PCa (Table 2) ranged from $9.8 \%$ (in primary lung cancer patients) to $25.7 \%$ (in primary rectal cancer patients). Similarly, the proportions of patients that died of primary cancers ranged from $16.4 \%$ (skin 
TABLE 2 | Baseline and prostate cancer characteristics of the 10 most common nonurological cancers prior to secondary prostate cancer.

\begin{tabular}{|c|c|c|c|c|c|c|c|}
\hline & $\begin{array}{l}\text { Median age at primary } \\
\text { cancer diagnosis (IQR) }\end{array}$ & $\begin{array}{l}\text { Median age at secondary } \\
\text { prostate cancer diagnosis } \\
\text { (IQR) }\end{array}$ & $\begin{array}{c}\text { Median PSA at } \\
\text { diagnosis in } \mathrm{ng} / \mathrm{ml} \\
\text { (IQR) }\end{array}$ & $\begin{array}{c}\text { RP vs. EBRT } \\
\text { treatment } \\
(\%)\end{array}$ & $\begin{array}{l}\text { Overall } \\
\text { deaths }\end{array}$ & $\begin{array}{l}\text { Died from secondary } \\
\text { prostate cancer }(\%)\end{array}$ & $\begin{array}{c}\text { Died from } \\
\text { primary } \\
\text { cancer }(\%)\end{array}$ \\
\hline $\begin{array}{l}\text { Skin cancer } \\
(n=3,871)\end{array}$ & $61(54-69)$ & $69(63-75)$ & $6.5(4.8-10.2)$ & 29.7 vs. 22.6 & 749 & 164 (21.9) & $123(16.4)$ \\
\hline $\begin{array}{l}\text { Rectal } \\
\text { cancer } \\
(n=798)\end{array}$ & $62(55-68)$ & $70(64-76)$ & $7.6(5.2-12.7)$ & 20.4 vs. 19.5 & 214 & $55(25.7)$ & $40(18.7)$ \\
\hline $\begin{array}{l}\text { Colon cancer } \\
(n=3,665)\end{array}$ & $65(58-71)$ & 72 (66-78) & $7.7(5.2-14.0)$ & 17.3 vs. 29.1 & 1,146 & 215 (18.8) & 213 (18.6) \\
\hline $\begin{array}{l}\text { Lymphoma } \\
(n=2,583)\end{array}$ & $62(55-69)$ & 69 (63-75) & $6.9(4.9-11.4)$ & 22.5 vs. 27.5 & 766 & $123(16.1)$ & 274 (35.8) \\
\hline $\begin{array}{l}\text { Leukemia } \\
(n=1,102)\end{array}$ & $63(56-70)$ & $69(64-75)$ & $6.8(4.9-11.1)$ & 22.2 vs. 23.6 & 340 & 45 (13.2) & 135 (31.4) \\
\hline $\begin{array}{l}\text { Pancreatic } \\
\text { cancer } \\
(n=118)\end{array}$ & 65 (60-70) & $70(65-74)$ & $7.8(5.1-13.5)$ & 14.4 vs. 26.3 & 34 & 6 (17.6) & $11(32.4)$ \\
\hline $\begin{array}{l}\text { Stomach } \\
\text { cancer } \\
(n=361)\end{array}$ & $64(58-71)$ & 71 (65-77) & $7.1(5.0-12.9)$ & 20.2 vs. 28.0 & 118 & $29(24.6)$ & $26(22.0)$ \\
\hline $\begin{array}{l}\text { Esophagus } \\
\text { cancer } \\
(n=219)\end{array}$ & 65 (59-69) & 70 (65-75) & $7.4(5.1-11.2)$ & 18.7 vs. 29.7 & 74 & $11(14.9)$ & $26(35.1)$ \\
\hline $\begin{array}{l}\text { Liver cancer } \\
(n=160)\end{array}$ & $61(56-67)$ & $66(61-71)$ & $7.8(5.8-12.8)$ & 11.3 vs. 34,4 & 52 & $11(21.2)$ & $26(50.0)$ \\
\hline $\begin{array}{l}\text { Lung cancer } \\
(N=1,328)\end{array}$ & 65 (59-71) & 71 (66-76) & $7.6(5.0-12.8)$ & 14.0 vs. 31.4 & 599 & $59(9.8)$ & 255 (42.6) \\
\hline $\begin{array}{l}\text { Overall } \\
(n=24,848)\end{array}$ & 63 (56-69) & $69(64-76)$ & $6.9(4.9-11.5)$ & 23.8 vs. 25.7 & 4,069 & 715 (17.6) & $1,122(27.6)$ \\
\hline
\end{tabular}

PSA, prostate-specific antigen; $R P$, radical prostatectomy; EBRT, external beam radiation therapy.

cancer) to $50.0 \%$ (liver cancer). Unfortunately, these cancer-specific rates could not be translated into Kaplan-Meier-derived actuarial estimates due to unavailable time to death.

\section{Survival Analyses After 1:4 Propensity Score Matching According to Time Interval Length Since Initial Cancer Diagnosis and Secondary PCa Diagnoses}

Time interval length since initial cancer and secondary $\mathrm{PCa}$ diagnoses was stratified into four groups between 7 and 36 $(n=6,659)$ vs. 37 and $60(n=4,759) \quad v s$. 61 and 120 $(n=7,289)$ vs. $\geq 121$ months $(n=6,141)$. In Kaplan-Meier plots (Figure 5) that addressed the comparison between secondary PCa diagnosed between 7 and 36 months after primary cancer diagnosis, relative to primary $\mathrm{PCa}$, the respective 10 -year OM rates were $47.4 \%$ vs. $30.4 \%$. These OM rates translated into a multivariable $\mathrm{HR}$ of 1.95 . The subsequent stratifications (37-60 vs. 61-120 vs. $\geq 121$ months) resulted in 10year OM rates in secondary PCa patients of $47.4 \%$ vs. $31.8 \%$, $45.1 \%$ vs. $32.3 \%$, and $44.0 \% \%$ vs. $35.2 \%$ months in primary $\mathrm{PCa}$ patients. The respective multivariable HR for 7-36 vs. 37-60 vs. 61-120 vs. $\geq 121$ months were $1.7,1.6$, and 1.3.

\section{DISCUSSION}

We hypothesized that secondary PCa patients will harbor less favorable disease characteristics in addition to exhibiting less favorable prognosis, relative to primary $\mathrm{PCa}$ patients. To test this hypothesis, we identified 24,848 secondary PCa patients and 531,732 primary PCa patients, for the purpose of comparisons. Here, secondary PCa patients were older than their primary PCa counterparts. On average, secondary $\mathrm{PCa}$ diagnosis (69 years) was made 6 years after primary cancer diagnosis (63 years). Moreover, age at diagnosis variability was also recorded according to primary cancer type in secondary PCa patients. The latter ranged from 66 (liver cancer) to 72 years (colon cancer). These observations are different from the more historical reports about secondary PCa. For example, in the study by Dinh et al., median age in patients with secondary PCa diagnosis was 73 , which is significantly older than in the current study (17). It may be postulated that a selection bias is operational regarding the age at secondary $\mathrm{PCa}$ diagnosis. The latter may be directly related to aggressiveness and mortality probability of the primary cancer diagnosis. Although such simplified explanation is attractive, several confounding variables may be operational. For example, patients with most aggressive cancers may be expected to be never be diagnosed with secondary PCa. Conversely, long-term survivors of highly aggressive primary cancer variants may still be diagnosed with secondary PCa. The latter may render generalizations about the effect of aggressive primary cancer on rates and ages at secondary $\mathrm{PCa}$ diagnosis virtually uninterpretable.

Less pronounced differences were recorded in PSA distributions of secondary and primary $\mathrm{PCa}$ patients, 
A

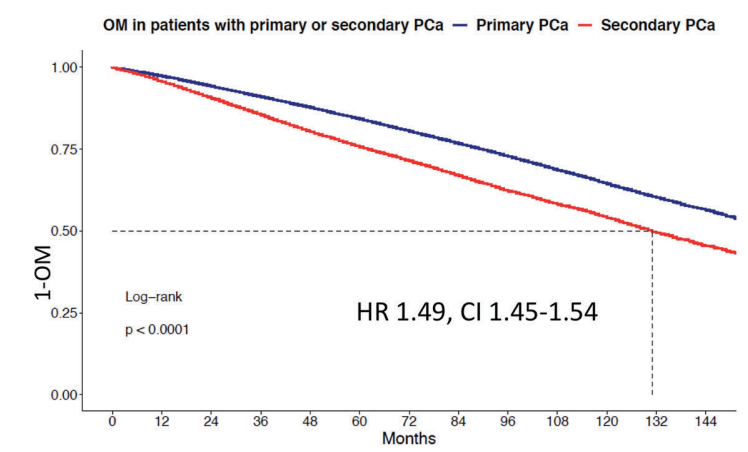
Number at risk

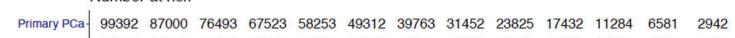
secondary PCa. \begin{tabular}{cccccccccccccc}
24848 & 21610 & 18556 & 15986 & 13408 & 11076 & 8812 & 6846 & 5077 & 3698 & 2349 & 1339 & 597 \\
\hline 0 & 12 & 24 & 36 & 48 & 60 & 72 & 84 & 96 & 108 & 120 & 132 & 144
\end{tabular}

C

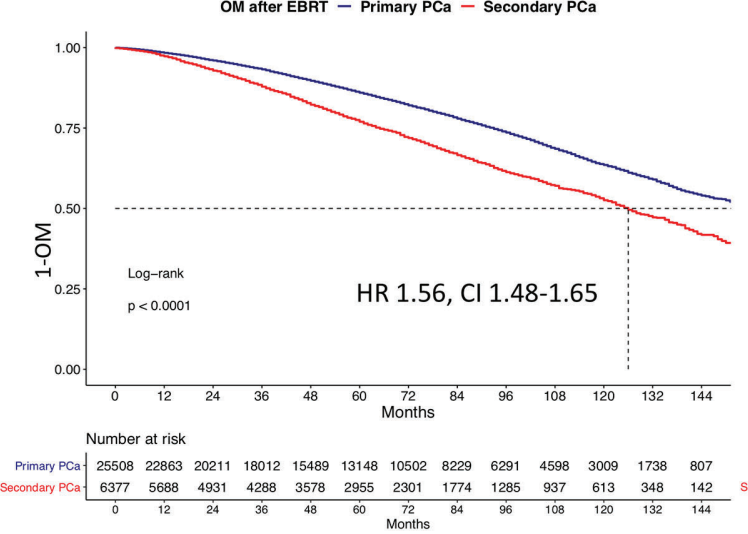

B

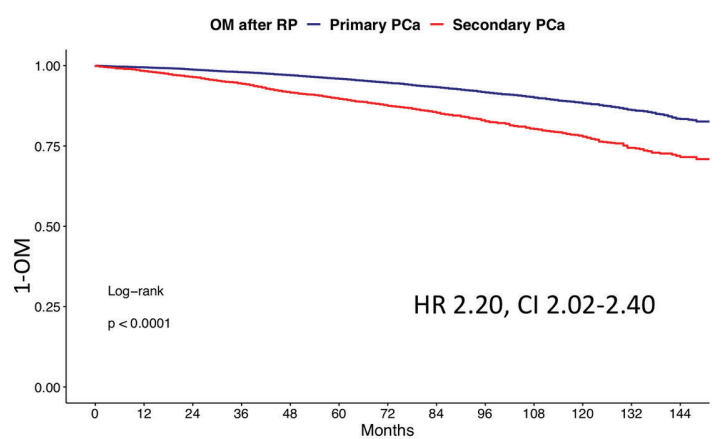

Number at risk

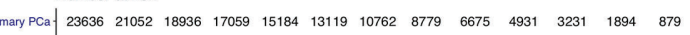
\begin{tabular}{ccccccccccccc|}
5909 & 5252 & 4672 & 4164 & 3634 & 3110 & 2537 & 2044 & 1539 & 1125 & 725 & 402 & 182 \\
\hline 0 & 12 & 24 & 36 & 48 & 60 & 72 & 84 & 96 & 108 & 120 & 132 & 144
\end{tabular}
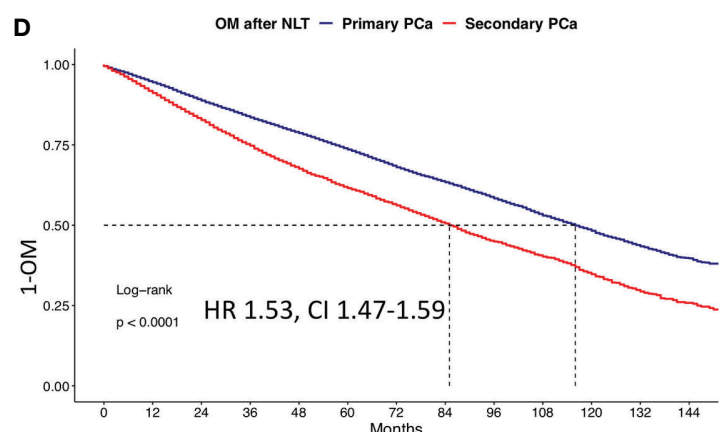

Number at risk

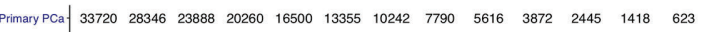

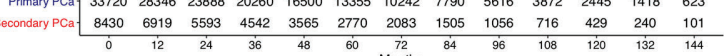

FIGURE 2 | Kaplan-Meier plots depicting overall mortality (OM) for primary and secondary prostate cancer for (A) the overall cohort, (B) patients treated with radical prostatectomy (RP), (C) patients treated with external beam radiation therapy (EBRT), and (D) no local treatment (NLT). HR, hazard ratio; Cl, confidence interval.

evidenced by respectively 6.9 (IQR $4.9-11.5$ ) vs. $6.6 \mathrm{ng} / \mathrm{ml}$ (IQR 4.8-10.6) PSA values at diagnoses. Additionally, small differences in PSA at diagnoses were recorded in secondary PCa patients, according to primary cancer type and ranged from 6.5 (skin cancer) to $7.8 \mathrm{ng} / \mathrm{ml}$ (pancreatic and liver cancers). Similarly, we also observed small differences in Gleason grade groups IV and V. Here, secondary PCa patients exhibited less favorable grade. This observation is in an agreement with previous publications, where secondary PCa patients also harbored higher rates of Gleason grade group IV/V $(18,19)$. Finally, no clinically meaningful differences were identified according to stage. Taken together, these data indicate that despite more advanced age and small disadvantage in PSA at diagnosis and PCa grade, secondary $\mathrm{PCa}$ patients do not exhibit crucial $\mathrm{PCa}$ characteristic differences at initial diagnosis. However, this interpretation may be biased and warrants methodologically more stringent analyses. This suspicion prompted the use of propensity score matching, according to age as well as patient and PCa characteristics. Moreover, we also applied additional multivariable adjustment in all subsequent survival analyses. The intent was to most thoroughly test for prognostic differences with strictest reduction of bias and/ or confounding.

In part 1 of the $\mathrm{OM}$ analyses, the propensity-matched comparisons addressed the entire cohort of secondary $\mathrm{PCa}$ patients, relative to all primary PCa controls. In part 2 of OM analyses, we examined the effect of primary and secondary $\mathrm{PCa}$ in respectively RP-, EBRT-, and NLT-treated patients. In the third part of the analyses, we sequentially compared secondary PCa patients, relative to their primary PCa counterparts, according to the type of primary malignancy diagnosed prior to secondary PCa. In the fourth part of analyses, we stratified the comparisons according to the length of the time interval between primary cancer and secondary PCa diagnoses.

In 1:4 matched survival analyses that addressed the entire secondary $\mathrm{PCa}$ population, relative to their primary $\mathrm{PCa}$ controls, we identified pronounced survival disadvantage in secondary PCa patients (10-year OM 46\% vs. 35.7\%). A similar absolute and relative magnitude of the survival disadvantage in secondary PCa patients was also recorded in subgroup analyses of RP-, EBRT-, and NLT-treated patients. In the third part of the analyses, we invariably recorded a survival disadvantage in all secondary PCa patients diagnosed with the 10 most common 
TABLE 3 | Univariable und multivariable Cox regression models after adjustment for PSA, socioeconomic status, Gleason grade group, and D'Amico risk stratification.

\begin{tabular}{|c|c|c|c|c|}
\hline & \multicolumn{2}{|c|}{ Univariable } & \multicolumn{2}{|c|}{ Multivariable } \\
\hline \multicolumn{5}{|l|}{ Cancers } \\
\hline All secondary prostate cancer & $1.49(1.45-1.54)$ & $<0.01$ & $1.51(1.47-1.55)$ & $<0.01$ \\
\hline Skin cancer & $1.10(1.02-1.20)$ & 0.02 & $1.16(1.07-1.26)$ & $<0.001$ \\
\hline Colon cancer & $1.22(1.15-1.31)$ & $<0.001$ & $1.15(1.08-1.23)$ & $<0.001$ \\
\hline Pancreatic cancer & $1.72(1.56-2.55)$ & $<0.01$ & $1.80(1.20-2.70)$ & $<0.01$ \\
\hline Stomach cancer & $1.73(1.40-2.14)$ & $<0.001$ & $1.92(1.54-2.38)$ & $<0.001$ \\
\hline Leukemia & $1.81(1.59-2.05)$ & $<0.001$ & $1.84(1.62-2.09)$ & $<0.001$ \\
\hline Esophagus cancer & $1.82(1.39-2.38)$ & $<0.001$ & $1.81(1.38-2.38)$ & $<0.001$ \\
\hline Lung cancer & $2.43(2.21-2.68)$ & $<0.001$ & $2.51(2.28-2.77)$ & $<0.001$ \\
\hline Liver cancer & $2.78(1.98-3.91)$ & $<0.001$ & $2.95(2.08-4.17)$ & $<0.001$ \\
\hline Secondary EBRT & $1.56(1.48-1.65)$ & $<0.001$ & $1.59(1.51-1.68)$ & $<0.001$ \\
\hline Primary prostate cancer and no local treatment & Ref & - & - & - \\
\hline Secondary no local treatment & $1.53(1.47-1.59)$ & $<0.001$ & $1.53(1.47-1.59)$ & $<0.001$ \\
\hline \multicolumn{5}{|l|}{ Time intervals } \\
\hline Primary prostate cancer & Ref & - & - & - \\
\hline Secondary cancer 7-36 months prior to prostate cancer & $1.92(1.83-2.02)$ & $<0.001$ & $1.95(1.85-2.05)$ & $<0.001$ \\
\hline Secondary cancer 37-60 months prior to prostate cancer & $1.77(1.67-1.88)$ & $<0.001$ & $1.74(1.64-1.85)$ & $<0.001$ \\
\hline Secondary cancer $61-120$ months prior to prostate cancer & $1.58(1.50-1.67)$ & $<0.001$ & $1.61(1.53-1.70)$ & $<0.001$ \\
\hline Secondary cancer $>120$ months prior to prostate cancer & $1.34(1.27-1.42)$ & $<0.001$ & $1.32(1.24-1.40)$ & $<0.001$ \\
\hline
\end{tabular}

$H R$, hazard ratio; $\mathrm{Cl}$, confidence interval.

A

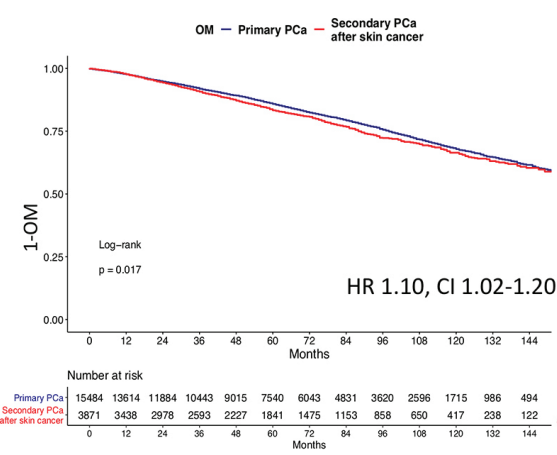

D

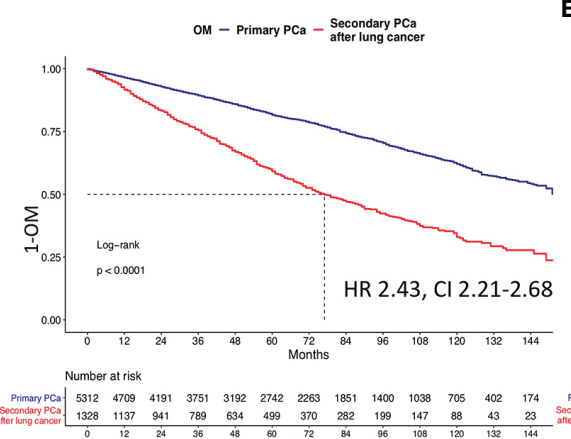

B

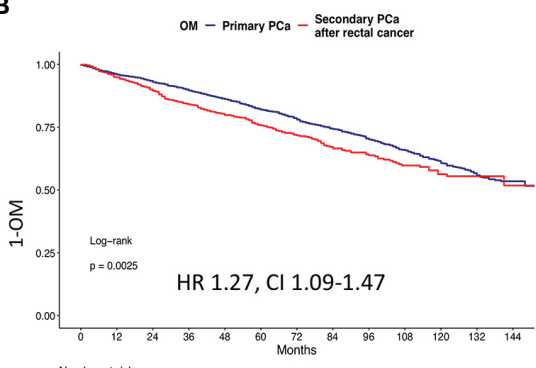

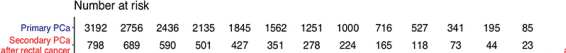

E

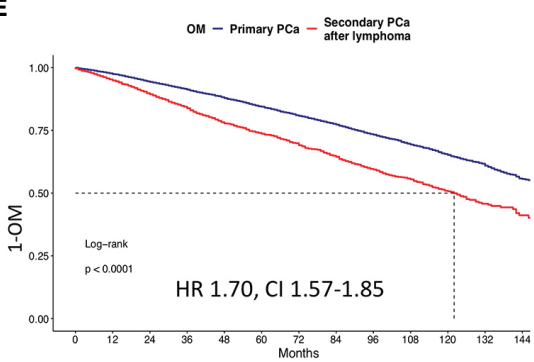

C

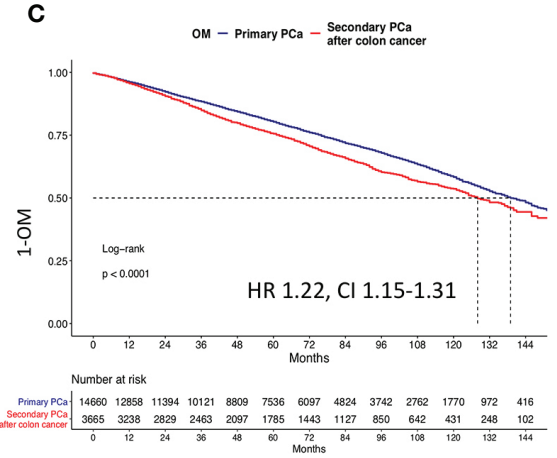

F

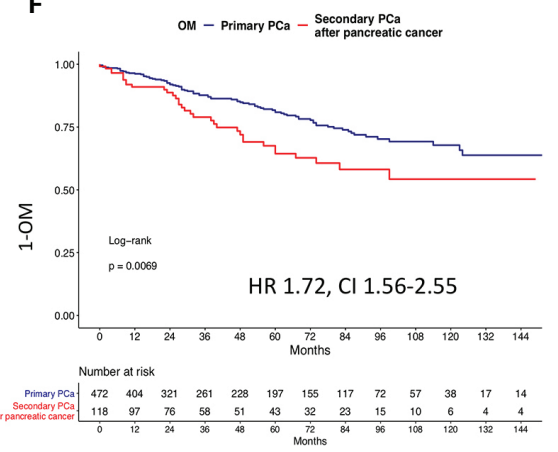

FIGURE 3 | Kaplan-Meier plots depicting overall mortality (OM) for primary and secondary prostate cancer after (A) primary skin cancer, (B) primary rectum cancer, (C) primary colon cancer, (D) primary lung cancer, (E) primary lymphoma, and (F) primary pancreatic cancer. HR, hazard ratio; Cl, confidence interval. 
A

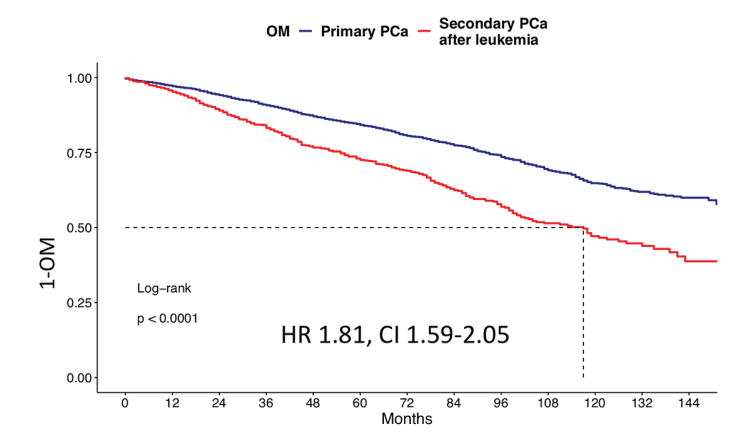

Number at risk

\begin{tabular}{l|lllllllllllll} 
Primary PCa & 4408 & 3780 & 3328 & 2937 & 2485 & 2102 & 1717 & 1357 & 1037 & 747 & 502 & 290 & 135
\end{tabular}

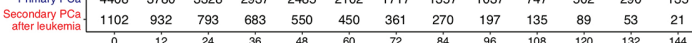

C

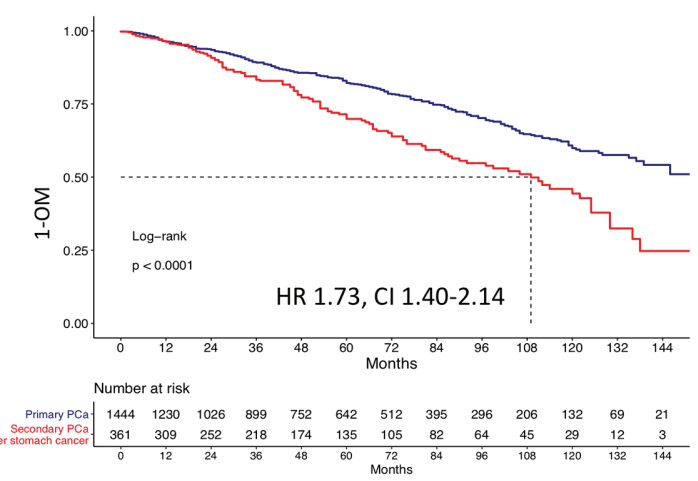

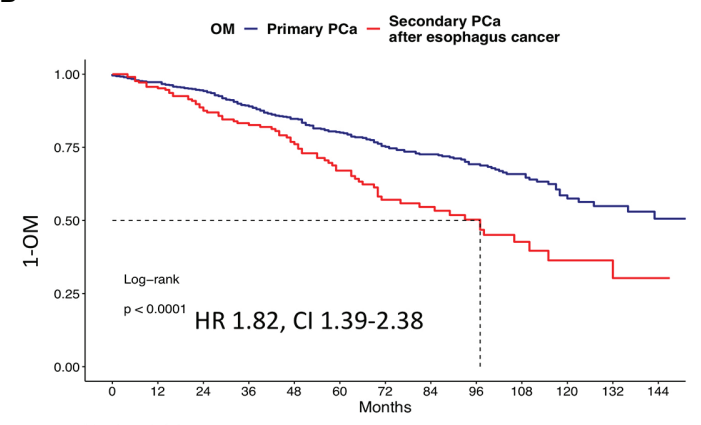

Number at risk

\begin{tabular}{l|lllllllllllll} 
Primary PCa & 876 & 771 & 665 & 560 & 451 & 372 & 284 & 221 & 163 & 109 & 54 & 33 & 20
\end{tabular}

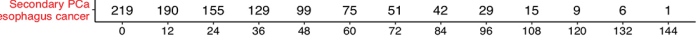

D

OM - Primary PCa - Secondary PCa

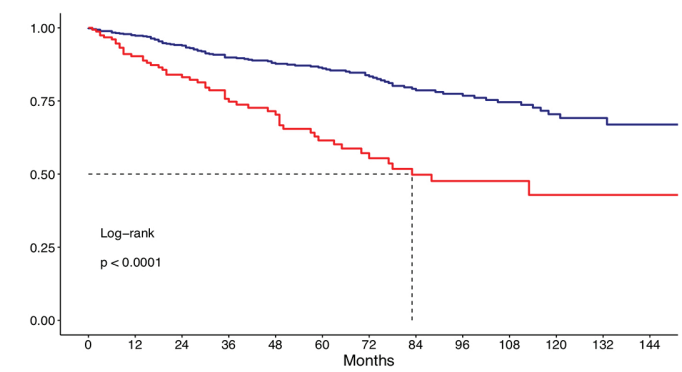

Number at risk

\begin{tabular}{c|ccccccccccccc}
$\begin{array}{c}\text { Primary PCa } \\
\text { Secondary PCa }\end{array}$ & 640 & 528 & 434 & 371 & 304 & 256 & 192 & 152 & 117 & 91 & 57 & 34 & 9
\end{tabular}

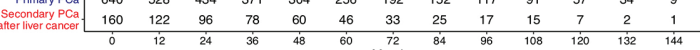

FIGURE 4 | Kaplan-Meier plots depicting overall mortality (OM) for primary and secondary prostate cancer after (A) primary leukemia, (B) primary liver cancer, (C) primary stomach cancer, and (D) primary esophagus cancer. HR, hazard ratio; Cl, confidence interval.

nonurological initial cancers (HRs: 1.1-2.8). These observations are consistent with previous findings. For example, Klippstein et al. also investigated a survival disadvantage (overall and cancer-specific survival) of 1,552 secondary PCa patients, relative to primary PCa patients (19). However, due to sample size limitations, no primary cancer-specific analyses could be conducted in these analyses and should be ideally performed in further multi-institutional analyses.

Taken together, the above findings indicate that despite apparently small to no differences in patient and/or PCa characteristics at baseline between secondary and primary PCa patients, very important survival disadvantages were applied to secondary PCa patients. This observation was made despite most stringent and methodologically strict statistical matching and multivariable adjustment. In consequence, the persistence of this disadvantage across therapy types suggest that secondary PCa patient harbor a prognostic disadvantage, relative to primary PCa patients, despite exhibiting almost the same baseline characteristics. The observed disadvantage applies across all primary cancer types and persists regardless of primary treatment type (RP and EBRT) and also after further multivariable adjustment for Gleason grade group and PSA. In consequence, the detrimental effect of secondary $\mathrm{PCa}$ appears robust and generalizable. The observation of Zhu et al. validates our hypothesis about the aggressiveness of primary cancer that may impact, as well as determine the natural history of treated secondary malignancies (24). The above findings, especially that with longer time interval between primary cancer and secondary PCa life expectancy approximates the life expectancy to primary $\mathrm{PCa}$, should be considered treatment decision making, when secondary $\mathrm{PCa}$ patients are counseled.

Finally, in analyses according to length of time interval between primary cancer and secondary PCa diagnoses, we observed that the survival disadvantage decreases with increasing length of time. This observation may indicate that in individuals in whom the time between initial and secondary cancer diagnoses is lengthy, the secondary PCa phenotype may be more comparable with primary PCa. Conversely, when the length of interval between primary cancer and secondary PCa is short, the phenotype might be more aggressive, as evidenced by greater survival disadvantage. We are the first to report this observation, which should be validated in other largescale databases.

Our observations imply that patients with secondary PCa should be given more careful consideration to eliminate the survival disadvantages that we recorded. Unfortunately, the nature of our data does not allow to identify whether the increase in $\mathrm{OM}$ in secondary PCa patients, relative to their primary PCa counterparts, 
A

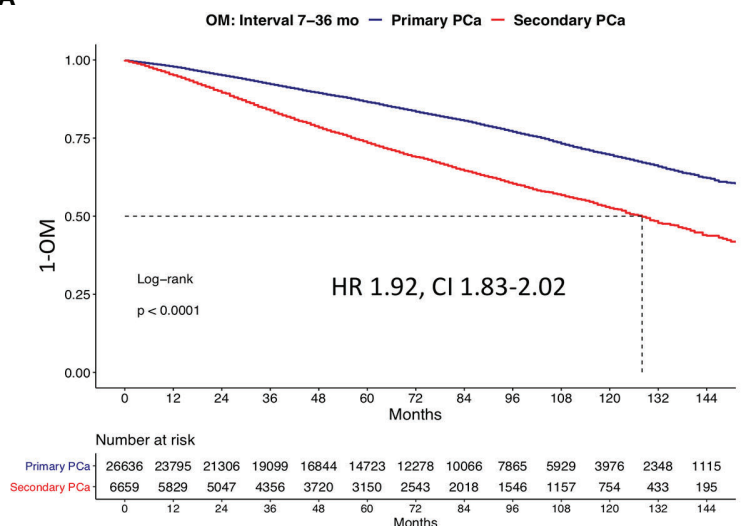

C

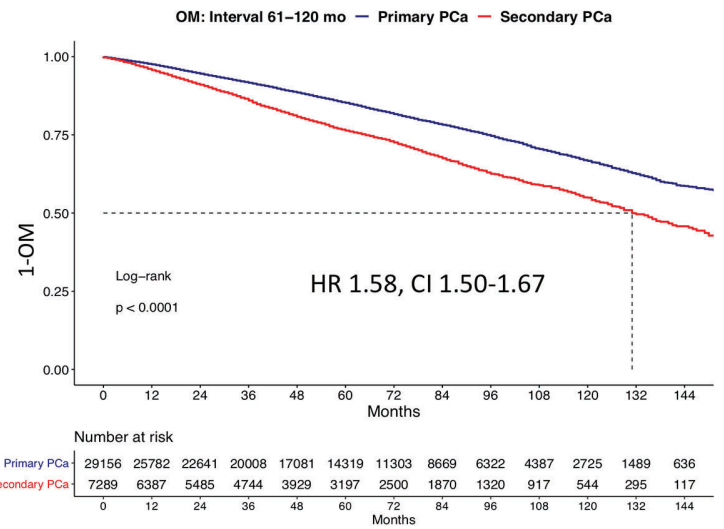

B

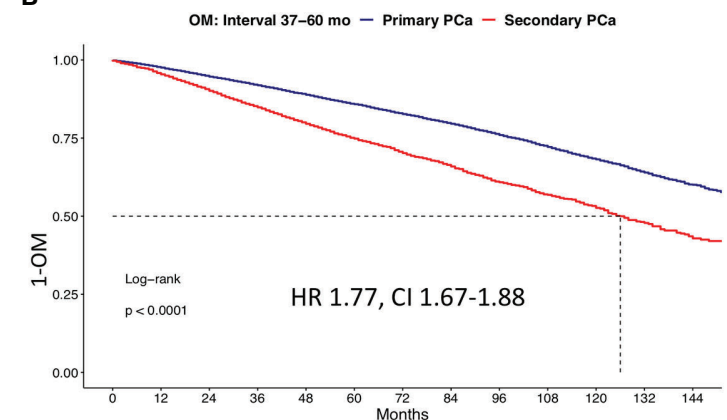

Number at risk

\begin{tabular}{l|lllllllllllll} 
Primary PCa- & 19036 & 17054 & 15236 & 13601 & 11936 & 10193 & 8437 & 6888 & 5267 & 3897 & 2613 & 1570 & 700
\end{tabular} \begin{tabular}{r|ccccccccccccc} 
Primary PCa & 19036 & 17054 & 15236 & 13601 & 11936 & 10193 & 8437 & 6888 & 5267 & 3897 & 2613 & 1570 & 700 \\
Secondary PCa & 4759 & 4202 & 3655 & 3164 & 2694 & 2227 & 1796 & 1427 & 1046 & 765 & 513 & 295 & 129 \\
\hline
\end{tabular}

D

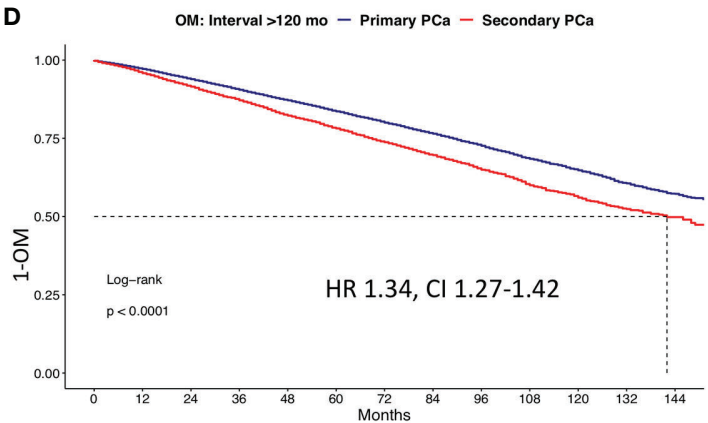
Number at risk

\begin{tabular}{l|lllllllllllll} 
Primary PCa & 24564 & 20860 & 17778 & 15400 & 12906 & 10730 & 8566 & 6806 & 5260 & 3848 & 2503 & 1453 & 732
\end{tabular} \begin{tabular}{cccccccccccccc} 
Primary PCa & 24564 & 20860 & 17778 & 15400 & 12906 & 10730 & 8566 & 6806 & 5260 & 3848 & 2503 & 1453 & 732 \\
\cline { 2 - 11 } & 6141 & 5191 & 4373 & 3722 & 3062 & 2497 & 1971 & 1533 & 1164 & 848 & 534 & 315 & 155 \\
\hline 0 & 12 & 24 & 36 & 48 & 60 & 72 & 84 & 96 & 108 & 120 & 132 & 144
\end{tabular}

FIGURE 5 | Kaplan Meier plots depicting overall mortality (OM) for primary and secondary prostate cancer according to the time interval between primary cancer and secondary prostate cancer at (A) 7-46 months, (B) 37-60 months, (C) 61-120 months, and (D) >120 months. HR, hazard ratio; Cl, confidence interval.

was related to the primary cancer or secondary PCa. In consequence, measures aimed at reducing this survival disadvantage of secondary PCa patients should not only focus on PCa treatments and follow-up but also on treatments and follow-up of their primary cancer. Finally, more detailed databases would allow to distinguish between mortality from primary or secondary cancer could help fine tuning further research and clinical management.

Our work has limitations and should be interpreted in the context of its retrospective and population-based design. Second, the nature of our data does not allow to define specific mortality time points to estimate Kaplan-Meier actuarial mortality rates. This limitation is shared with all previous publications focusing on secondary cancers, after specific primary cancers in large-scale databases (24-26). Limited stage and grade information was available for each of the 10 examined primary cancers and matching could not be performed for PSA and Gleason grade group without losing secondary PCa patients. Finally, important variables such as performance status and comorbidities are not available in the SEER database (27). These also contribute to OM rates but could neither be addressed in the current study or in previous analyses (24-26).

\section{DATA AVAILABILITY STATEMENT}

The raw data supporting the conclusions of this article will be made available by the authors, without undue reservation.

\section{ETHICS STATEMENT}

Ethical review and approval was not required for the study on human participants in accordance with the local legislation and institutional requirements. Written informed consent for participation was not required for this study in accordance with the national legislation and the institutional requirements.

\section{AUTHOR CONTRIBUTIONS}

Conceptualization: MW, LN, CR, FC, and PK. Methodology: MW and ZT. Formal analysis and investigation: MW, CW, and ZT. Writing (original draft preparation): MW, LN, FC, and PK. Writing (review and editing): FS, ABr, DT, MG, ABe, FR, and FC. Supervision: FS, ABr, FR, FC, and PK. All authors contributed to the article and approved the submitted version. 


\section{REFERENCES}

1. Bray F, Ferlay J, Soerjomataram I, Siegel RL, Torre LA, Jemal A. Global Cancer Statistics 2018: GLOBOCAN Estimates of Incidence and Mortality Worldwide for 36 Cancers in 185 Countries. CA Cancer J Clin (2018) 68 (6):394-424. doi: 10.3322/caac.21492

2. Ferlay J, Colombet M, Soerjomataram I, Dyba T, Randi G, Bettio M, et al. Cancer Incidence and Mortality Patterns in Europe: Estimates for 40 Countries and 25 Major Cancers in 2018. Eur J Cancer (2018) 103:356-87. doi: 10.1016/j.ejca.2018.07.005

3. Siegel RL, Miller KD, Jemal A. Cancer Statistics, 2019. CA Cancer J Clin (2019) 69(1):7-34. doi: 10.3322/caac.21551

4. Wenzel M, Würnschimmel C, Chierigo F, Mori K, Tian Z, Terrone C, et al. Pattern of Biopsy Gleason Grade Group $5(4+5$ vs $5+4$ vs $5+5)$ Predicts Survival After Radical Prostatectomy or External Beam Radiation Therapy. Eur Urol Focus (2021) 28:S2405-4569(21)00117-6. doi: 10.1016/j.euf.2021.04.011

5. Wenzel M, Würnschimmel C, Chierigo F, Tian Z, Shariat SF, Terrone C, et al. Assessment of the Optimal Number of Positive Biopsy Cores to Discriminate Between Cancer-Specific Mortality in High-Risk Versus Very High-Risk Prostate Cancer Patients. Prostate (2021) 81(14):1055-63. doi: 10.1002/ pros. 24202

6. Würnschimmel C, Wenzel M, Wang N, Tian Z, Karakiewicz PI, Graefen M, et al. Long-Term Overall Survival of Radical Prostatectomy Patients is Often Superior to the General Population: A Comparison Using Life-Table Data. Prostate (2021) 81(11):785-93. doi: 10.1002/pros.24176

7. Nocera L, Wenzel M, Collà Ruvolo C, Würnschimmel C, Tian Z, Gandaglia G, et al. The Effect of Race/Ethnicity on Active Treatment Rates Among Septuagenarian or Older Low Risk Prostate Cancer Patients. Urol Oncol (2021) 12:S1078-1439(21)00161-7. doi: 10.1016/j.urolonc.2021.04.004

8. Wenzel M, Würnschimmel C, Nocera L, Collà Ruvolo C, Tian Z, Shariat SF, et al. The Effect of Lymph Node Dissection on Cancer-Specific Survival in Salvage Radical Prostatectomy Patients. Prostate (2021) 81(6):339-46. doi: $10.1002 /$ pros. 24112

9. Würnschimmel C, Wenzel M, Collà Ruvolo C, Nocera L, Tian Z, Saad F, et al. Life Expectancy in Metastatic Prostate Cancer Patients According to Racial/ Ethnic Groups. Int J Urol (2021) 28(8):862-9. doi: 10.1111/iju.14595

10. Soerjomataram I, Coebergh JW. Epidemiology of Multiple Primary Cancers. Methods Mol Biol (2009) 471:85-105. doi: 10.1007/978-1-59745-416-2_5

11. Donin N, Filson C, Drakaki A, Tan H-J, Castillo A, Kwan L, et al. Risk of Second Primary Malignancies Among Cancer Survivors in the United States, 1992 Through 2008. Cancer (2016) 122(19):3075-86. doi: 10.1002/cncr.30164

12. Ng AK, Travis LB. Subsequent Malignant Neoplasms in Cancer Survivors. Cancer J (2008) 14(6):429-34. doi: 10.1097/PPO.0b013e31818d8779

13. Van Hemelrijck M, Drevin L, Holmberg L, Garmo H, Adolfsson J, Stattin P. Primary Cancers Before and After Prostate Cancer Diagnosis. Cancer (2012) 118(24):6207-16. doi: 10.1002/cncr.27672

14. Kok DEG, van de Schans S a M, Liu L, Kampman E, Coebergh JWW, Kiemeney L a LM, et al. Risk of Prostate Cancer Among Cancer Survivors in the Netherlands. Cancer Epidemiol (2013) 37(2):140-5. doi: 10.1016/j.canep. 2012.11.004

15. Moot AR, Polglase A, Giles GG, Garson OM, Thursfield V, Gunter D. Men With Colorectal Cancer Are Predisposed to Prostate Cancer. ANZ J Surg (2003) 73(5):289-93. doi: 10.1046/j.1445-2197.2003.t01-1-02621.x

16. Kawakami S, Fukui I, Yonese J, Ueda T, Ohno Y, Tsuzuki M, et al. Multiple Primary Malignant Neoplasms Associated With Prostate Cancer in 312 Consecutive Cases. Urol Int (1997) 59(4):243-7. doi: 10.1159/000283072
17. Dinh KT, Mahal BA, Ziehr DR, Muralidhar V, Chen Y-W, Viswanathan VB, et al. Risk of Prostate Cancer Mortality in Men With a History of Prior Cancer. BJU Int (2016) 117(6B):E20-8. doi: 10.1111/bju.13144

18. Mirabeau-Beale K, Chen M-H, D'Amico AV. Prior-Cancer Diagnosis in Men With Nonmetastatic Prostate Cancer and the Risk of Prostate-Cancer-Specific and All-Cause Mortality. ISRN Oncol (2014) 2014:736163. doi: 10.1155/2014/ 736163

19. Klippenstein P, Schlomm T, von Amsberg G, Beyer B, Pompe RS, Michl U, et al. Prostate Cancer Prognosis in Men With Other Malignancies Prior to Radical Prostatectomy. Urol Oncol (2019) 37(9):575.e1-7. doi: 10.1016/ j.urolonc.2019.04.007

20. Wenzel M, Würnschimmel C, Nocera L, Ruvolo CC, Tian Z, Saad F, et al. The Effect of Primary Urological Cancers on Survival in Men With Secondary Prostate Cancer. Prostate (2021) 81(15):1149-58. doi: 10.1002/pros.24209

21. Kamath GR, Kim MK, Taioli E. Risk of Primary Neuroendocrine Pancreatic Tumor After a First Primary Cancer: A US Population-Based Study. Pancreas (2019) 48(2):161-8. doi: 10.1097/MPA.0000000000001232

22. Clift AK, Drymousis P, Al-Nahhas A, Wasan H, Martin J, Holm S, et al. Incidence of Second Primary Malignancies in Patients With Neuroendocrine Tumours. Neuroendocrinology (2015) 102(1-2):26-32. doi: 10.1159/ 000381716

23. RCT. R: A Language and Environment for Statistical Computing (2017) Available at: https://wwwr-projectorg2017.

24. Zhu K, Lin R, Zhang Z, Chen H, Rao X. Impact of Prior Cancer History on the Survival of Patients With Larynx Cancer. BMC Cancer (2020) 20(1):1137. doi: 10.1186/s12885-020-07634-2

25. Laccetti AL, Pruitt SL, Xuan L, Halm EA, Gerber DE. Prior Cancer Does Not Adversely Affect Survival in Locally Advanced Lung Cancer: A National SEER-Medicare Analysis. Lung Cancer (2016) 98:106-13. doi: 10.1016/ j.lungcan.2016.05.029

26. He C, Zhang Y, Cai Z, Lin X. Effect of Prior Cancer on Survival Outcomes for Patients With Pancreatic Adenocarcinoma: A Propensity Score Analysis. BMC Cancer (2019) 19(1):509. doi: 10.1186/s12885-019-5744-8

27. Wenzel M, Würnschimmel C, Chierigo F, Tian Z, Shariat SF, Terrone C, et al. Non-Cancer Mortality in Elderly Prostate Cancer Patients Treated With Combination of Radical Prostatectomy and External Beam Radiation Therapy. Prostate (2021) 81(11):728-35. doi: 10.1002/pros.24169

Conflict of Interest: The authors declare that the research was conducted in the absence of any commercial or financial relationships that could be construed as a potential conflict of interest.

Publisher's Note: All claims expressed in this article are solely those of the authors and do not necessarily represent those of their affiliated organizations, or those of the publisher, the editors and the reviewers. Any product that may be evaluated in this article, or claim that may be made by its manufacturer, is not guaranteed or endorsed by the publisher.

Copyright (c) 2021 Wenzel, Nocera, Würnschimmel, Collà Ruvolo, Tian, Saad, Briganti, Tilki, Graefen, Becker, Roos, Chun and Karakiewicz. This is an openaccess article distributed under the terms of the Creative Commons Attribution License (CC BY). The use, distribution or reproduction in other forums is permitted, provided the original author(s) and the copyright owner(s) are credited and that the original publication in this journal is cited, in accordance with accepted academic practice. No use, distribution or reproduction is permitted which does not comply with these terms. 